University of Nebraska - Lincoln

DigitalCommons@University of Nebraska - Lincoln

\title{
A parallel computing approach to fast geostatistical areal interpolation
}

\author{
Qingfeng Guan \\ University of Nebraska - Lincoln, qguan2@unl.edu \\ Phaedon Kyriakidis \\ University of California - Santa Barbara, phaedon@geog.ucsb.edu \\ Michael Goodchild \\ University of California - Santa Barbara, good@geog.ucsb.edu
}

Follow this and additional works at: https://digitalcommons.unl.edu/geographyfacpub

Part of the Geographic Information Sciences Commons

Guan, Qingfeng; Kyriakidis, Phaedon; and Goodchild, Michael, "A parallel computing approach to fast geostatistical areal interpolation" (2011). Geography Faculty Publications. 34.

https://digitalcommons.unl.edu/geographyfacpub/34

This Article is brought to you for free and open access by the Geography Program (SNR) at DigitalCommons@University of Nebraska - Lincoln. It has been accepted for inclusion in Geography Faculty Publications by an authorized administrator of DigitalCommons@University of Nebraska - Lincoln. 


\title{
A parallel computing approach to fast geostatistical areal interpolation
}

\author{
Qingfeng Guan, ${ }^{1}$ Phaedon C. Kyriakidis, ${ }^{2}$ and Michael F. Goodchild ${ }^{2}$ \\ 1. Center for Advanced Land Management Information Technologies, \\ School of Natural Resources, University of Nebraska-Lincoln, Lincoln, NE, USA \\ 2. Department of Geography, University of California Santa Barbara, Santa Barbara, CA, USA \\ Corresponding author - Q. Guan, email qguan2@unl.edu
}

\begin{abstract}
Areal interpolation is the procedure of using known attribute values at a set of (source) areal units to predict unknown attribute values at another set of (target) units. Geostatistical areal interpolation employs spatial prediction algorithms, that is, variants of Kriging, which explicitly incorporate spatial autocorrelation and scale differences between source and target units in the interpolation endeavor. When all the available source measurements are used for interpolation, that is, when a global search neighborhood is adopted, geostatistical areal interpolation is extremely computationally intensive. Interpolation in this case requires huge memory space and massive computing power, even with the dramatic improvement introduced by the spectral algorithms developed by Kyriakidis et al. (2005. Improving spatial data interoperability using geostatistical support-to-support interpolation. In: Proceedings of geoComputation. Ann Arbor, MI: University of Michigan) and Liu et al. (2006. Calculation of average covariance using fast Fourier transform (FFT). Menlo Park, CA: Stanford Center for Reservoir Forecasting, Petroleum Engineering Department, Stanford University) based on the fast Fourier transform (FFT). In this study, a parallel FFT-based geostatistical areal interpolation algorithm was developed to tackle the computational challenge of such problems. The algorithm includes three parallel processes: (1) the computation of source-to-source and source-to-target covariance matrices by means of FFT; (2) the QR factorization of the source-to-source covariance matrix; and (3) the computation of source-to-target weights via Kriging, and the subsequent computation of predicted attribute values for the target supports. Experiments with real-world datasets (i.e., predicting population densities of watersheds from population densities of counties in the Eastern Time Zone and in the continental United States) showed that the parallel algorithm drastically reduced the computing time to a practical length that is feasible for actual spatial analysis applications, and achieved fairly high speed-ups and efficiencies. Experiments also showed the algorithm scaled reasonably well as the number of processors increased and as the problem size increased.
\end{abstract}

Keywords: parallel computing, geostatistics, fast Fourier transform, Kriging, areal interpolation

\section{Introduction}

\subsection{Areal interpolation}

The geospatial data used for current GIS/GeoComputation-enabled spatial analysis are mainly produced by associating a set of attributes (either quantitative measurements 
or qualitative descriptions) with a set of spatial objects, or spatial basis defined by Goodchild et al. (1993), for example, points, lines, and areas in vector map data, or cells/pixels in raster grids. In general, an interpolation problem involves a set of spatial objects with their attribute $z$ known, that is, the source; and another set of spatial objects with their attribute $z$ unknown, that is, the target. The goal is to predict the unknown attribute $z$ of the target objects using the source objects and their attribute values. A common variant of spatial interpolation is point interpolation, which predicts the attribute values at target locations using a set of points over space with known attribute values. This work focuses on another type of spatial interpolation: areal interpolation. Areal interpolation is the transfer of attribute values from one existing spatial partition to another. The unknown (target) attribute values associated with a particular partition (target zones or supports) are predicted from a set of known (source) attribute data available on a different partition (source zones or supports), where supports are defined as spatial domains informed by attribute values, for example, lines in one dimension or zones (polygons) in two dimensions (Kyriakidis et al. 2005). As for the types of areal attributes, we follow the terminology of Goodchild and Lam (1980), by referring to extensive attributes as areal totals, for example, populations of counties; and intensive attributes as areal averages, for example, population densities of counties.

Haining (2003) distinguished two categories of areal interpolation approaches: cartographic and statistical. In the first category, geometrical characteristics of the source and target supports are treated as the main factor in spatial prediction. Goodchild and Lam (1980) discussed the properties of an areal interpolation technique that uses the areas of intersection between source and target polygons as weights. In the second category of areal interpolation, based on statistical methods, values of a dependent attribute at the target zones are predicted from auxiliary data (predictors) available at those zones based on a regression model linking the dependent and the auxiliary data at the source zones. A third category of areal interpolation approaches could be identified, invoking principles of geostatistics (see, e.g., Kyriakidis et al. 2005). In that category, target attribute values are predicted using different forms of Kriging and a model of spatial correlation while accounting for the support differences between source and target supports. Since geostatistical interpolation methods are formulated in a probabilistic setting, the prediction error variance can be estimated along with the prediction. Furthermore, Kyriakidis et al. (2005) proved that geostatistical areal interpolation provides mass-preserving target predictions. More recently, Kyriakidis and Goodchild (under review) proved that popular cartographic areal interpolation methods, such as interpolation via proportional area weighting and dasymetric mapping, can be regarded as particular cases of geostatistical areal interpolation.

Despite its many advantages over cartographic approaches, geostatistical areal interpolation has a vital disadvantage that greatly obstructs its usage in time-sensitive (realtime or near real-time) real-world applications: the extreme computational intensity. Especially when all available source measurements are involved in interpolation, a situation also known as global interpolation, huge memory space and massive computing power are required. Enormous computing time is therefore required, which is usually far beyond being feasible (examples will be given in the following sections). Global interpolation is needed when spatial attribute interaction has a large range compared with the size of supports and the extent of the study region, for example, international trade. In some special cases, one may be interested in interactions between attribute values at the boundary and the interior, which calls for a near-global interpolation (see Yoo and Kyriakidis 2008). The goal of this article is to employ parallel computing technology to improve the 
performance of such geostatistical areal interpolation algorithms and reduce their computing time to a practical length for real-world spatial analysis applications.

In fact, parallelizing interpolation algorithms is not a new topic in GIScience and GeoComputation. Armstrong and coworkers have worked extensively on parallelizing point interpolation on a variety of parallel computing platforms, including massive parallel computers and the computational Grid (Armstrong and Marciano 1993, 1994, 1995, 1996, 1997, Wang and Armstrong 2003). Furthermore, during the past decade, many studies have parallelized Kriging-based point interpolation. Most of these studies parallelize the interpolation process by dividing the output grid into sub-grids and distributing them to multiple processors (Gajraj et al. 1997, Kerry and Hawick 1998, Morrison 2000, Hawick et al. 2003, Pedelty et al. 2003, Rossini et al. 2007). However, the computational demand for areal interpolation using Kriging systems is much higher than that for point interpolation. To construct a Kriging system for areal interpolation, the covariance matrices for the source and target supports (e.g., polygons) have to be computed. The entries of these matrices require enormous time to compute due to the repeated evaluation of spatial integrals (Chilès and Delfiner 1999). Fortunately, the computation for each entry in the covariance matrix is independent of that for other entries, meaning that the support-to-support covariance computation can be parallelized. Nevertheless, little work has been done specifically on parallelizing geostatistical areal interpolation, and this article contributes to this effort.

\subsection{Geostatistical areal interpolation}

Geostatistical areal interpolation assumes there exists a latent point field $\{z(u), u \in D\}$ of an attribute $Z$, where $z(u)$ is the unobserved point attribute value at location $u$, and $D$ denotes the study domain. The $k$ th source datum $z\left(s_{k}\right)$ is then assumed to be an integrated measurement of unknown point $z$-values within that non-point (e.g., areal) support:

$$
z\left(s_{k}\right)=\int_{u \in D} g_{k}(u) z(u) d u, \quad k=1,2, \ldots, K
$$

where $g_{k}(u)$ denotes a known sampling function for the $k$ th support that represents the contribution of each point $u \in D$ to the corresponding source support datum $z\left(s_{k}\right)$. For extensive variables,

$$
g_{k}(u)=\left\{\begin{array}{l}
1 \text { if } u \in s_{k} \\
0 \text { if } u \notin s_{k}
\end{array}\right.
$$

For intensive variables,

$$
g_{k}(u)=\left\{\begin{array}{c}
\frac{1}{\left|s_{k}\right|} \text { if } u \in s_{k} \\
0 \text { if } u \notin s_{k}
\end{array}\right.
$$

where $\left|s_{k}\right|$ is the measure (e.g., area of a polygon in 2D) of the $k$ th support.

In a geostatistical setting, the unknown point attribute field $\{z(u), u \in D\}$ is viewed as one out of many possible realizations of a random field model $\{Z(u), u \in D\}$, where $Z(u)$ denotes the random variable $(\mathrm{RV})$ at location $u$. Under intrinsic and second-order station- 
arity, the mean of the point random field model is considered constant, and the semivariogram $\gamma$ and the covariogram $\sigma$ between any two RVs $Z(u)$ and $Z\left(u^{\prime}\right)$ are only functions of the vector $h=u-u^{\prime}$ separating the two points $u$ and $u^{\prime}$, that is, $\gamma_{z}\left(u-u^{\prime}\right)=\gamma_{z}(h)$ and $\sigma_{z}(u-$ $\left.u^{\prime}\right)=\sigma_{z}(h)$.

Under the above formulation, the covariance between two areal support RVs $Z\left(s_{k}\right)$ and $Z\left(s_{k^{\prime}}\right)$ can be written as (see, e.g., Goovaerts 1997)

$$
\sigma_{z}\left(s_{k^{\prime}}, s_{k^{\prime}}\right)=\operatorname{Cov}\left\{Z\left(s_{k}\right), Z\left(s_{k^{\prime}}\right)\right\}=\int_{u \in D} \int_{u^{\prime} \in D} g_{k}(u) \sigma_{z}\left(u-u^{\prime}\right) g_{k^{\prime}}\left(u^{\prime}\right) d u^{\prime} d u
$$

In real-world applications, the study domain is discretized into a grid of points to represent the unobserved field, and the $k$ th source datum is then the discrete sum of the unknown point attribute values over that support:

$$
z\left(s_{k}\right) \approx \sum_{i=1}^{N} g_{k}\left(u_{i}\right) z\left(u_{i}\right), \quad k=1,2, \ldots, K
$$

where $N$ is the number of points comprising the discretization grid.

Similarly, the covariance between two areal support RVs $Z\left(s_{k}\right)$ and $Z\left(s_{k^{\prime}}\right)$ can be written as the discrete weighted sum of point covariances:

$$
\sigma_{z}\left(s_{k}, s_{k^{\prime}}\right)=\operatorname{Cov}\left\{Z\left(s_{k}\right), Z\left(s_{k^{\prime}}\right)\right\} \approx \sum_{i=1}^{N} \sum_{j=1}^{N} g_{k}\left(u_{i}\right) \sigma_{z}\left(u_{i}-u_{j}\right) g_{k^{\prime}}\left(u_{j}\right) \text { or } \sigma_{k k^{\prime}}=g_{k}^{T} C g_{k^{\prime}}
$$

where $\sigma_{k k^{\prime}}$ is the covariance between the support $k$ and $k^{\prime}, g_{k}$ a vector of sampling functions of all the points for the $k$ th support, and $g_{k}^{T}$ the transpose of $g_{k} ; C$ is a $(N \times N)$ covariance matrix between all pairs of discretization points and $g_{k^{\prime}}$ a vector of sampling functions of all the points for the $k^{\prime}$ th support.

Consider there are $K$ source supports and $P$ unknown target supports within the study domain $D$. The corresponding simple Kriging system for predicting the unknown attribute value $z\left(t_{q}\right)$ for target support $t_{q}$ can be written as

$$
\sum_{k^{\prime}=1}^{K} w_{p}\left(s_{k^{\prime}}\right) \sigma_{z}\left(s_{k^{\prime}}, s_{k^{\prime}}\right)=\sigma_{z}\left(t_{p^{\prime}} s_{k}\right), \quad k=1,2, \ldots, K \text { or } \sum_{\mathrm{ss}} w_{\mathrm{st}}=\sigma_{\mathrm{st}}
$$

where $\sum_{\mathrm{sS}}$ is the source-to-source (STS) covariance matrix between all $(K \times K)$ pairs of source supports, $\sigma_{\text {st }}$ the source-to-target (STT) covariance vector between all $K$ source supports and the target support $t_{p}$, and $w_{\text {st }}$ the vector of weights given to the source attribute values for predicting the unknown target value $z\left(t_{p}\right)$. Note again that global, as opposed to local, Kriging is considered in this work, meaning that all $K$ source data are used to predict the attribute value of each target support. The parallel algorithm presented in this work, however, can be readily applied to the cases of local Kriging, too.

Once the STS and STT covariance matrices are computed, the STT weights $w_{\text {st }}$ can be calculated by solving Equation (7). To solve such an $A x=b$ problem, factorization (or decomposition) of matrix $A$ (i.e., the STS covariance matrix $\sum_{\mathrm{ss}}$ in this case) is usually performed, such as LU, Cholesky, or QR factorization. To accommodate the errors in the STS covariance matrix that might be introduced by previous computation procedures, this 
study uses the QR factorization to solve the Kriging system and obtain the least squares solution of weights. Given an $m \times n$ full rank matrix $A$ with $m \geq n$, the QR factorization decomposes it into an $m \times n$ orthogonal matrix $Q$ (i.e., $Q^{\mathrm{T}} Q=I$ ) and an $n \times n$ upper-triangular matrix $R$, so that $A=Q R$. The pseudo-code for $Q R$ factorization is as follows (Karniadakis and Kirby 2003):

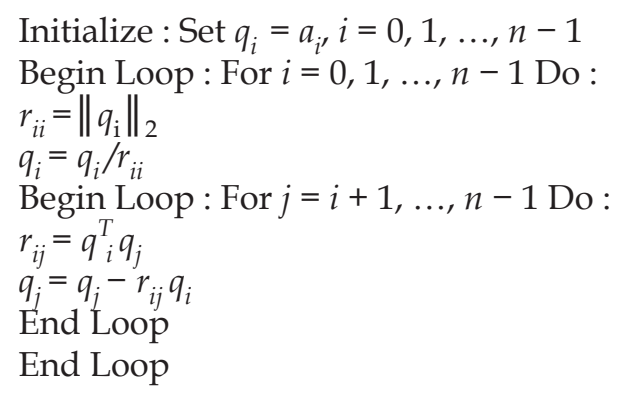

where $a_{i}$ is the $i$ th column of matrix $A ; q_{i}$ the $i$ th column of matrix $Q ;\left\|q_{i}\right\|_{2}$ the $L_{2}$ norm of $q_{i}$, which is defined as $\sqrt{\sum_{k=1}^{m} q_{k i}^{2}}$; and $r_{i j}$ the entry at the junction of the $i$ th row and $j$ th column of matrix $R$.

After QR factorization, Equation (7) can be transformed as follows:

$$
\sum_{\mathrm{ss}} w_{\mathrm{st}}=\sigma_{\mathrm{st}} \rightarrow Q R w_{\mathrm{st}}=\sigma_{\mathrm{st}} \rightarrow R w_{\mathrm{st}}=Q^{\mathrm{T}} \sigma_{\mathrm{st}}
$$

Once $Q^{\mathrm{T}} \sigma_{\text {st }}$ is computed, Equation (8) can be solved using the back-substitution method since $R$ is an upper-triangular matrix. Then the target value can be derived simply by multiplying the weights $w_{\text {st }}$ with the source values.

A typical geostatistical areal interpolation includes four steps:

Step 1: Discretization of source and target supports with a regular raster (point attribute values not known, just their location). Note that support discretization with irregularly placed points is also possible, but it is not pursued in this work.

Step 2: $\quad$ Computation of support-to-support covariance matrices as integrals from a given point covariance model (i.e., a stationary covariogram model):

(a) between all source supports,

(b) between all source and target supports.

Step 3: Use of a Kriging system with computed covariance matrices to derive weights for interpolation.

Step 4: Interpolated values computed as linear combinations of the Kriging weights and the source data values.

Without adopting any parallel technology, the standard geostatistical areal interpolation (see, e.g., Haining 2003) is highly computationally intensive, due to the need for multiple evaluations of covariance integrals over arbitrary-shaped regions. Kyriakidis et al. (2005) developed a fast integration algorithm for computing the covariance between any two 
supports using fast Fourier transform (FFT), which is much more efficient than the standard approach. The FFT-based approach will be briefly reviewed in the next section before describing the novel contribution of this article.

\subsection{FFT-based geostatistical areal interpolation and its computational complexity}

According to Kyriakidis et al. (2005), the covariance between two supports can be computed by multiplying the forward FFT of the extended sampling function vector of the first support, the forward FFT of the first row of the extended point covariance matrix, and the forward FFT of the extended sampling function vector of the second support. Extension of the various matrices is required for invoking the FFT processing. In a 2D case, an $N x \times N y$ matrix is usually extended to a $2 N x \times 2 N y$ matrix, where $N x$ and $N y$ denote the numbers of columns and rows of a matrix:

$$
\sigma_{z}\left(s_{k}, s_{k^{\prime}}\right)=\operatorname{Cov}\left\{Z\left(s_{k}\right), Z\left(s_{k^{\prime}}\right)\right\}=\operatorname{FFT}\left(\overline{g_{k}}\right) \otimes \operatorname{FFT}(\bar{C}(1,:)) \otimes \operatorname{FFT}\left(\overline{g_{k^{\prime}}}\right)
$$

where $\overline{g_{k}}$ and $\overline{g_{k^{\prime}}}$ are extended sampling function vectors of the supports, $\bar{C}(1,:)$ the first row of the extended point covariance matrix, and $\otimes$ denotes element-wise or Hadamard product.

This FFT-based geostatistical areal interpolation algorithm significantly reduces the computing time when compared with the traditional approach (i.e., computing supportto- support covariance matrices by integrating the given point covariance values over the supports' sampling function vectors). For an artificial test dataset (20 source supports and 25 target supports), the algorithm reportedly produced approximately an 80 -fold time decrease over the traditional approach.

However, the speed-up was achieved using a relatively simple artificial dataset. When it comes to real-world applications, this FFT-based algorithm is still computationally intensive, especially when dealing with highly dense discretization grids and large numbers of source and target supports. A Matlab-implemented interpolation application with the northern California population data on a $500 \times 500$ discretization grid requires 900-second computing time on a PC. Real-world geospatial datasets are usually large and complex, leading to an exponential increase in memory demand and computing time. Thus, single-processor computers might not be sufficient for applications that involve datasets covering wide areas or require high accuracies (i.e., dense discretization) in combination with a need for near real-time response. Most of the computational intensity comes from the FFT processing, which requires extensive amount of memory space and CPU time. In fact, the FFT processes in the algorithm account for more than $80 \%$ of the total CPU time (Liu et al. 2006). Suppose there are $K$ source supports and $P$ target supports, this algorithm will require $K+P+1$ FFT processes, where the extra one is the FFT on the first row of the extended point covariance matrix. Assuming the computational complexity of the FFT algorithm to be used is $O(N \log N)$ (Frigo 1999), where $N$ is the number of discretization points, and $N$ is much larger than $K$ and $P$, the overall computational complexity for the FFTs will be $O((K+P) \times N \log N)$.

Liu et al. (2006) developed a hybrid algorithm based on Kyriakidis et al.'s (2005) full FFT-based algorithm to calculate support-to-support covariances. Instead of performing the forward FFT on both of the supports' extended sampling function vectors and the first row of the extended point covariance matrix, Liu et al.'s (2006) algorithm only performs the forward FFT on the first support's extended sampling function vector and the first row of the extended point covariance matrix. The inverse FFT is performed on the 
product of these two FFTs to produce the support-to-point covariance matrix for the first support. Then the support-to-support covariance can be computed via the traditional approach, that is, integrating or averaging the support-to-point covariance values over the second support's sampling function vector and this is achieved via a straightforward matrix multiplication. The computational complexity of the hybrid approach is $O(2 \mathrm{~K} \times \mathrm{N}$ $\log N)$, whereas the complexity of the full FFT-based approach is $O((K+P) \times N \log N)$. More importantly, the full FFT-based approach requires enormous amount of run-time memory space to accommodate the fast Fourier transformed extended sampling function matrices of all source and target supports. When double-precision floating-point numbers (each requires 8 bytes in memory) are used to store the matrices, $(K+P) \times 2 N x \times 2 N y \times 8$ bytes are needed. On the other hand, the hybrid approach only needs to store the supportto-point covariance matrix for a particular source support at a time, which is merely $2 N x$ $\times 2 N y \times 8$ bytes. Once the support-to-support covariance values that involve this source support are computed, the memory for this support-to-point covariance matrix can be released for the next source support. Given the usually large volume of source and target datasets, and the high density of discretization points, the fast Fourier transformed sampling function matrices and covariance matrices are often too large to fit in the core memory of a processor, which may lead to memory allocation failure and has become one of the major factors of degradation in performance for the FFT-based areal interpolation. One of our case studies (see Section 3) was to predict the population densities of watersheds (1633 polygons) in the Eastern Time Zone from the population densities of counties (2248 polygons) using a discretization grid of $1333 \times 917$. The full FFT-based approach would require a memory space of 141.38 gigabytes (GB) at once just for the fast Fourier transformed sampling function matrices, while the hybrid approach only needs 37.3 megabytes (MB) at each iteration for the support-to-point covariance matrix. Apparently, the hybrid algorithm requires much less run-time memory space than the full algorithm does. We therefore chose Liu et al.'s (2006) algorithm as the base of our parallel algorithm.

\section{Parallelization and implementation}

\subsection{Parallelizability analysis}

Since the FFT processing for a particular support is independent of that for other supports, and the covariance computation between any two supports is also independent of the computation between any other two supports (see Equation (6)), this step (i.e., step 2 of the general algorithm described in Section 1.2) can be easily parallelized. Liu et al.'s (2006) hybrid algorithm implies that to compute the STT covariance matrix, it is not necessary to perform FFT on both the source supports and the target supports, but only on the source supports, which will significantly reduce both the memory space needed for the fast Fourier transformed sampling function matrices and the CPU time for FFT processing. Therefore, this step can be parallelized over the source supports by assigning subsets of source supports to multiple processors, so that each processor only computes the support-to-point covariances for a particular group of source supports via FFTs. If the processor also holds the sampling function vectors of all source and target supports, then a portion of the STS covariance matrix and a portion of the STT covariance matrix can be computed locally on the processor (the gray parts in Figure 1).

Furthermore, once the global ${ }^{1}$ STS and STT covariance matrices are computed, the Kriging system for estimating the STT weights and the computation of the attribute value 


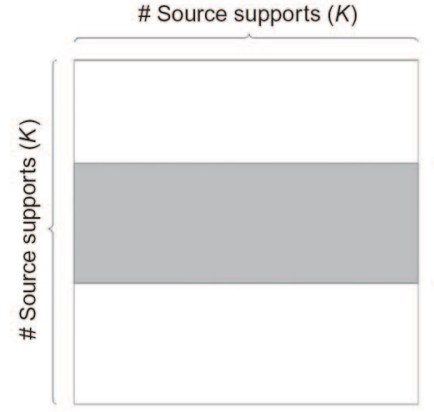

(a)

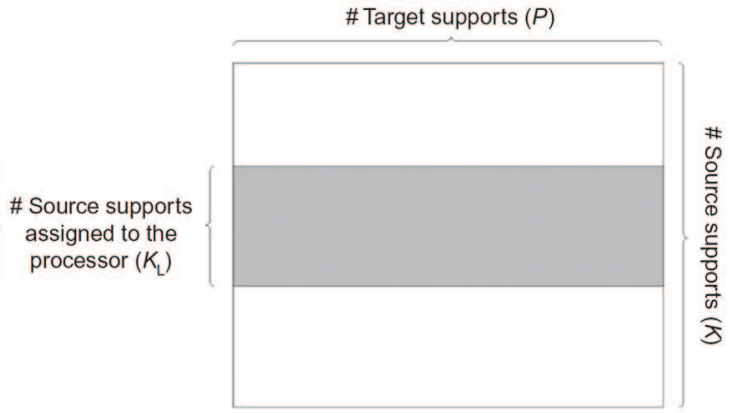

(b)

Figure 1. Covariance matrices held by a processor; the gray parts are computed locally on the processor (block task mapping). (a) The source-to-source covariance matrix held by a processor. (b) The source-to-target covariance matrix held by a processor.

for a particular target support is also independent of that for other target supports (see Equation (7)). Thus, these steps (i.e., steps 3 and 4 of the general algorithm described in Section 1.2) can be parallelized over the target supports. Each processor is assigned with a subset of the target supports, extracts the corresponding covariance values from the global STT covariance matrix to construct the Kriging system (Figure 2), and computes the STT weights, then computes the attribute values for the local ${ }^{2}$ target supports.

Two issues are critical for the above parallelization. First, the point covariances are fully defined by the covariogram model (i.e., the nugget, sills, ranges, and anisotropy angles) and the discretization scheme (i.e., the grid's node spacing or cell size, and the location of the grid origin). In other words, given a particular covariogram model and a discretization scheme, the point covariance matrix should always be the same no matter on which processor it is computed. Second, given a particular discretization scheme, the discretization process should always produce the same sampling function vectors for the supports no matter on which processor it is executed.

In addition, due to the usual large number of source supports in areal interpolation, the global STS covariance matrix is often massive in size and requires a large amount of computing time to factorize. A parallelization of the factorization process is therefore beneficial. As discussed in Section 1.2, the QR factorization is an iterative process. At each iteration, each column of the matrix $Q$ (which has the same dimensions as that of the STS

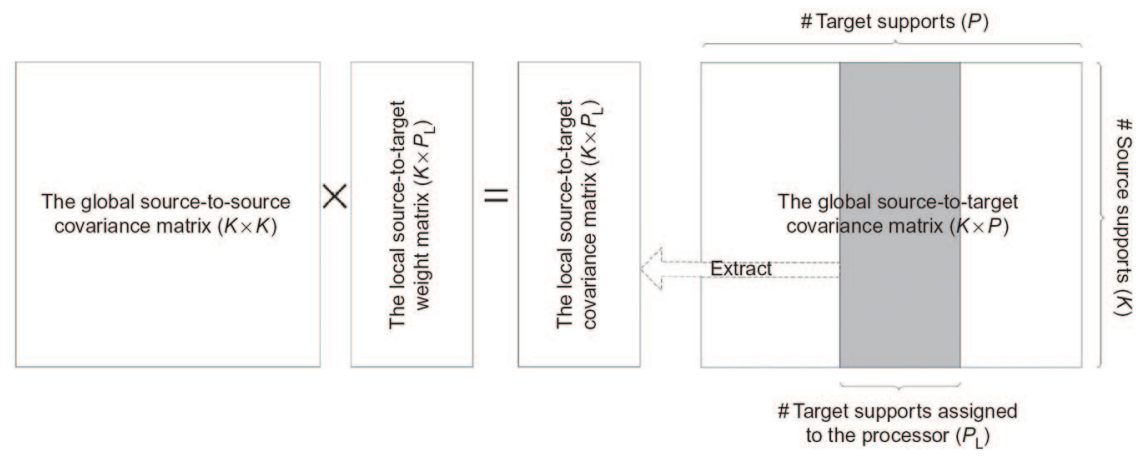

Figure 2. Kriging system constructed locally on a processor (block task mapping). 
covariance matrix) is updated independently. Thus, a QR factorization can be parallelized over the columns of $Q$ (Grama et al. 2003, Karniadakis and Kirby 2003).

\subsection{Parallel algorithm design}

In this study, the parallelization of the FFT-based geostatistical areal interpolation algorithm includes three parallel processes: (1) the FFT processing for the source supports and the computation of the STS and STT covariance matrices; (2) the QR factorization of the STS covariance matrix; and (3) the computation of the STT weights via Kriging and the consequent computation of attribute values for the target supports.

The detailed work flow is described as follows (Figure 3):

(1) Broadcasting the dataset, covariogram model, and discretization scheme

The geometry of both the source and target supports, the attribute data of the source supports, and a user-defined covariogram model are read onto the master processor. Based on the user-defined discretization options and the spatial information obtained from the source and target supports, a discretization scheme is determined by the master processor. All these data are then broadcasted from the master processor to all the processors engaged in the computation.

(2) Constructing the point covariance matrix and performing FFT

On each processor, the covariogram model and discretization scheme are used to construct the first row of the extended point covariance matrix. Then a forward FFT is performed on it. These processes are performed on all processors instead of performing them on the master processor and broadcasting the resultant vector to other processors, because transferring the resultant vector through the interconnecting network linking the processors is much less efficient than computing it locally on the processors, given that the size of the vector is usually large and the transfer rate of the network is low.

(3) Discretizing source and target supports

The geometry of the source and target supports is used to build the sampling function matrices for all source and target supports based on the discretization scheme obtained in step 1. Extremely small supports (i.e., supports into which no discretization point falls) will be ignored and their corresponding entries will be removed from the sampling function matrices. Attribute values corresponding to such small supports will also be removed from the attribute vector as well. Note this step is also performed on all processors, for the same reason given above. As discussed in Section 2.1, all processors will generate identical fast Fourier transformed extended point covariance map and sampling function matrices, since they hold the same covariogram model, discretization scheme, and support geometrical data.

(4) Task mapping

On each processor, two task maps are built based on the user-defined task-mapping option for both the source and target supports. A task map contains as many entries as the number of the processors, and each entry contains a processor's ID and the IDs of supports that are assigned to the process (Table 1). Note that since all processors use the same task-mapping scheme, task maps should be identical across processors.

The task-mapping scheme determines which task will be executed on which processor. It divides the task set into a certain number (usually the number of 
1250 Guan et al. in International Journal of Geographical Information SCience 25 (2011)

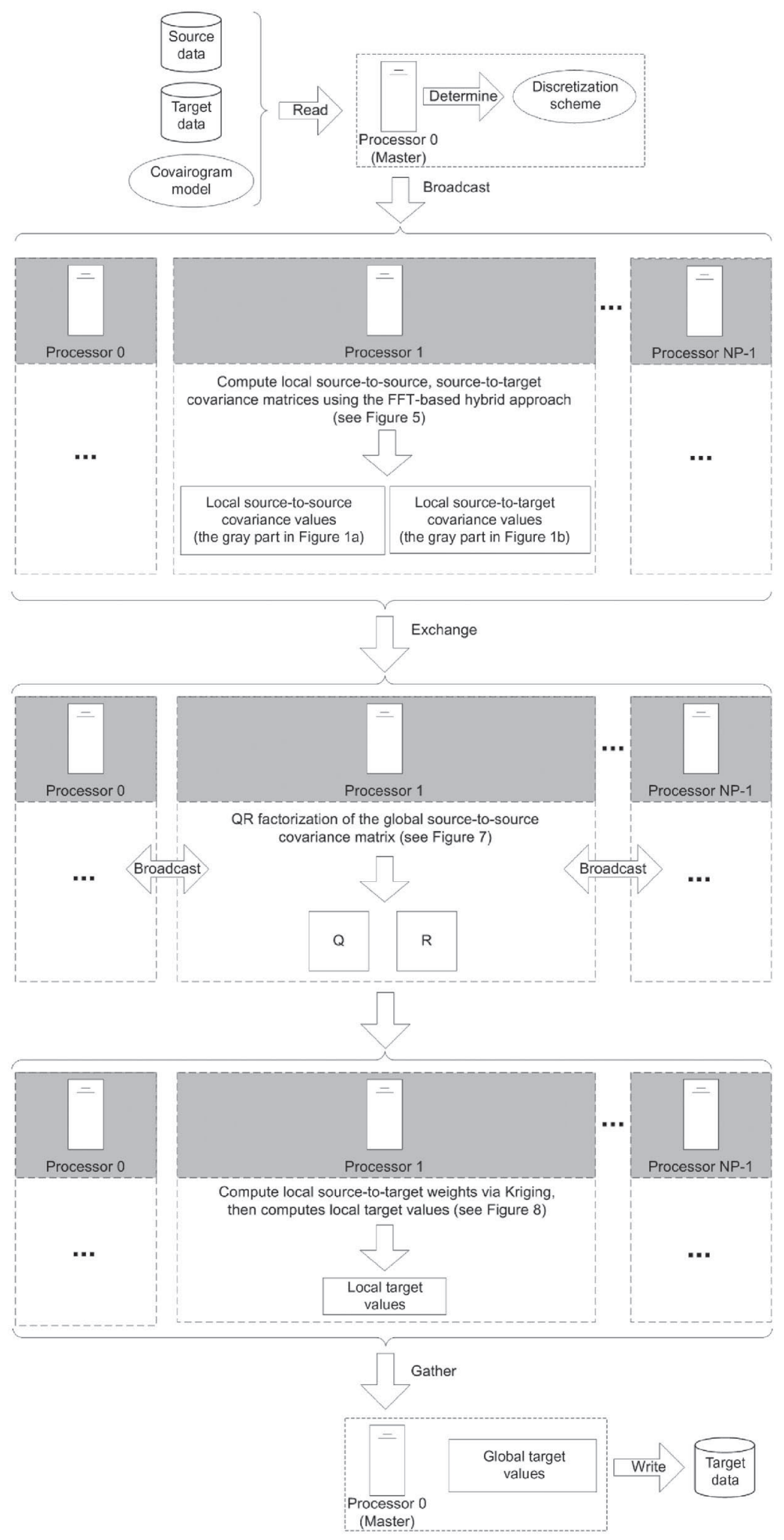

Figure 3. Three parallel processes for the FFT-based areal interpolation. 
Table 1. An example of task map produced by a block task-mapping scheme.

\begin{tabular}{ll}
\hline Processor ID & Support IDs \\
\hline 0 & $0,1,2, \ldots, i$ \\
1 & $\mathrm{i}+1, \mathrm{i}+2, \ldots, j$ \\
$\ldots$ & $\ldots$ \\
$\mathrm{NP}-1$ & $1,1+1, \ldots, k$ \\
\hline
\end{tabular}

processors, or NP) of task subsets and assigned them to processors. Several taskmapping schemes can be used, for example, block, cyclic, block-cyclic, cyclicblock, and random. Given a vector of tasks (i.e., supports in this case), the block task-mapping scheme partitions the vector into NP equally sized segments and assigns each segment to a processor, so that each processor will have a set of consecutive tasks to perform locally (Figure 4a). In contrast, the cyclic mapping scheme starts from the first task of the task vector, and repeatedly assigns a task per processor until the end of the task vector (Figure $4 \mathrm{~b}$ ). The block-cyclic and cyclic-block mapping schemes are simply the combinations of the block and cyclic schemes. The random mapping scheme also repeatedly assigns a task per processor, but chooses the task randomly from the task set instead of following the order of the task vector as the cyclic mapping scheme does (Figure 4c). Since all processors use the same random seed that is generated and broadcasted from the master processor, they will generate the same random number sequence, hence identical task maps.

Note that the dimensions of the sampling function vectors for all supports should be identical because they are built based on the same discretization scheme, thus the computational complexity of the FFT performed on the sampling function vector should be the same, that is, $O(N \log N)$, across supports. Also, the computational complexity of solving the Kriging system and computing the attribute value is the same for each target support. Since all task-mapping schemes used in this study distribute approximately the same number of source supports and target supports to processors, the workload of support-tosupport covariance computation and target attribute prediction for each processor should be the very similar. In other words, the workload is evenly distributed onto processors.

(5) Computing local STS and STT covariance values via FFT (first parallel process, Figure 5) According to its assignments in the source task map constructed in the previous step, each processor extracts the sampling function vectors from the source sampling function matrix consecutively, one at a time. For a source support to be processed locally, a forward FFT is first applied on the extended sampling function vector, and the resultant matrix is multiplied with the fast Fourier transformed extended point covariance matrix, which is obtained in step 2. An inverse FFT is then applied on the multiplication resultant matrix to produce the supportto-point covariance matrix for this particular source support. By using the traditional approach for calculating support-to-support covariances, that is, integrating the first support's support-to-point covariance values over the sampling functions of the second support, the covariance values between this source support and other source supports and all target supports are computed locally. 


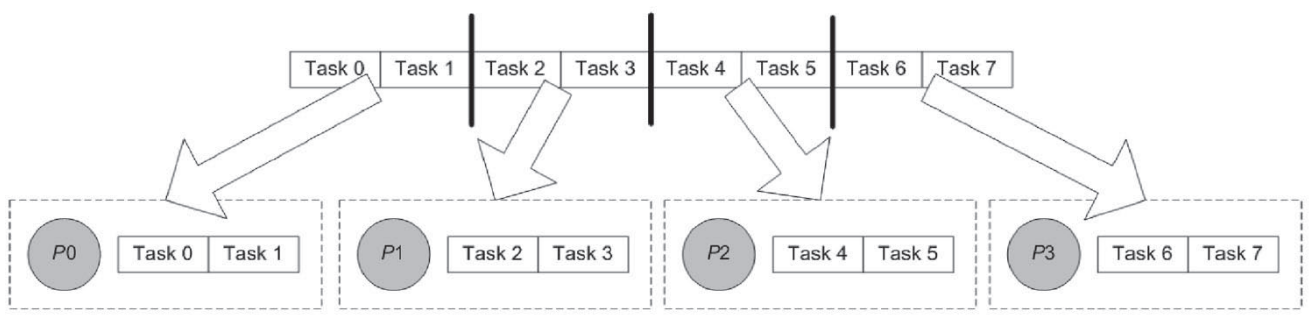

(a)

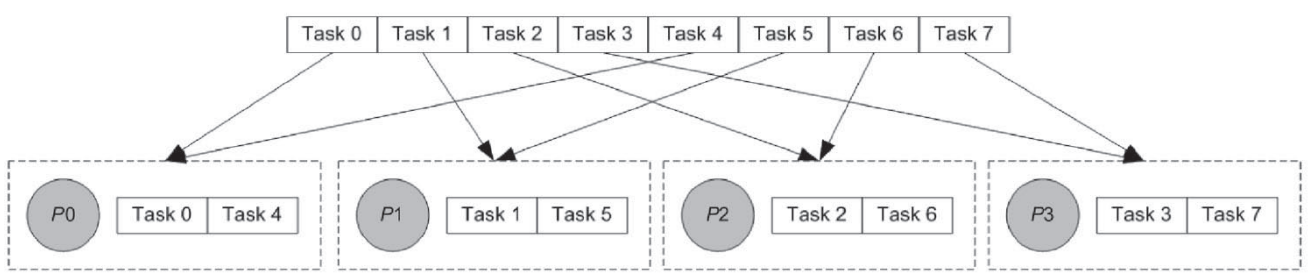

(b)

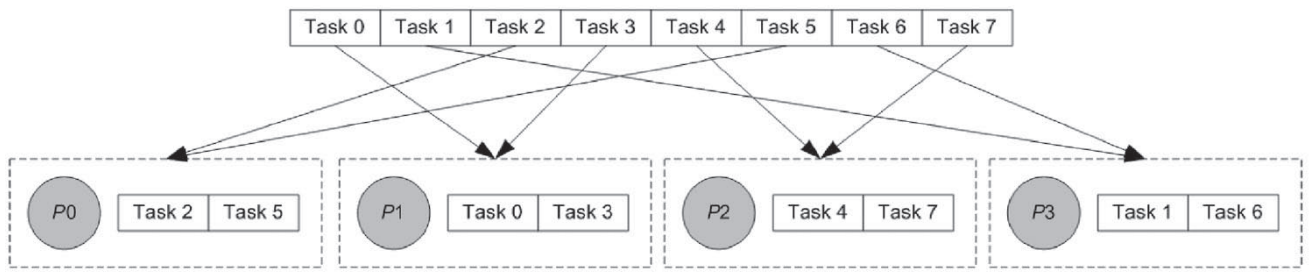

(c)

Figure 4. Task-mapping schemes for eight tasks and four processors. (a) Block task mapping; (b) cyclic task mapping; (c) random task mapping.

Once the STS and STT covariance values for a local source support are computed, the support-to- point covariance matrix for this source support is eliminated to release the memory space. By repeating this process on all local source supports, a processor computes a part of the global STS covariance matrix and a part of the global STT covariance matrix (the gray parts in Figure 1). Note that since the STS covariance matrix is a symmetric matrix along the diagonal, that is, $\operatorname{Cov}\left(s_{k^{\prime}} s_{k^{\prime}}\right)=$ $\operatorname{Cov}\left(s_{k^{\prime}} s_{k}\right)$, only one side (upper or lower) of the diagonal of the covariance matrix needs to be computed (in this study, the upper part is computed, Figure 6), and the other part can be obtained by simply copying the corresponding values from the computed part.

(6) Exchanging covariance values and completing global STS and STT covariance matrices The processors exchange the values that have been computed locally to form the global STS and STT covariance matrices. Again, since the STS covariance matrix is symmetric along the diagonal, only one side of the diagonal (upper part in this study) needs to be exchanged, and the resultant matrix is an upper diagonal matrix. The global STS covariance matrix is then completed by copying the values from the upper side to the mirror entries in the lower part of the matrix. 


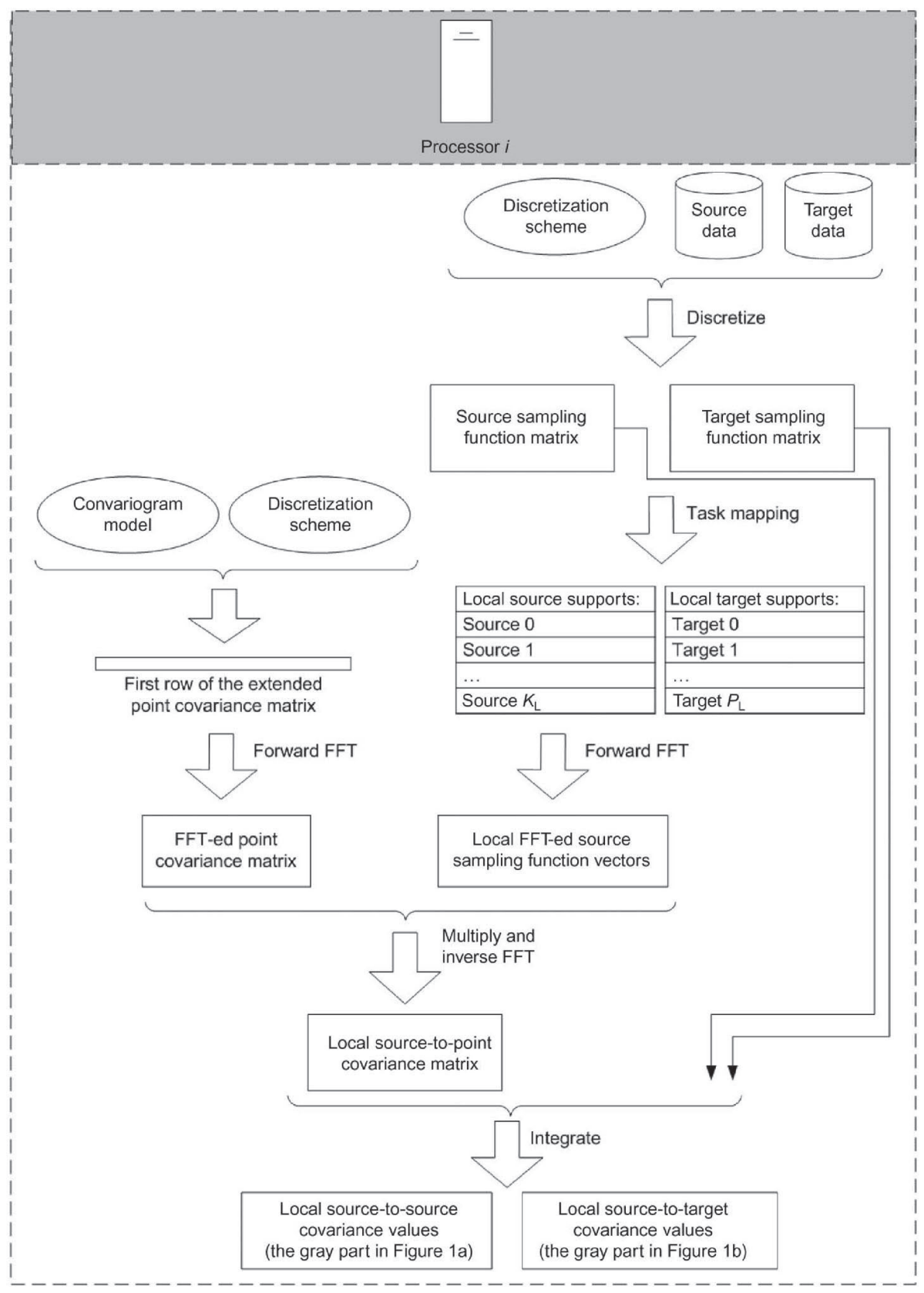

Figure 5. Detailed workflow of a processor for the first parallel process.

(7) Performing QR factorization on the global STS covariance matrix (second parallel process, Figure 7)

After step 6, each processor has a copy of the global STS covariance matrix in memory. A parallel QR factorization is then performed. The cyclic task-mapping scheme is used over the columns of the matrix $Q$ (see Section 1.2) to evenly distribute the workloads onto processors. At each iteration, each processor updates 


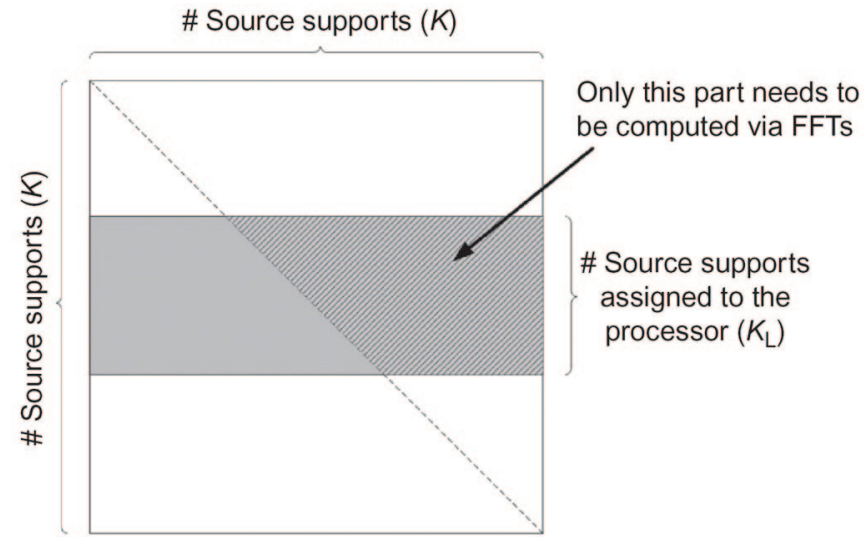

Figure 6. Part of the global STS covariance matrix is computed locally via FFTs.

a particular group of the columns of $Q$, and a completed column (that will not be updated again and will be used at the next iteration by all processors) is broadcasted to all processors. In the end, all processors have an identical QR factorization (including an orthogonal matrix $Q$ and an upper-triangular matrix $R$ ) of the STS covariance matrix in memory. Since the parallel algorithms for QR factor-

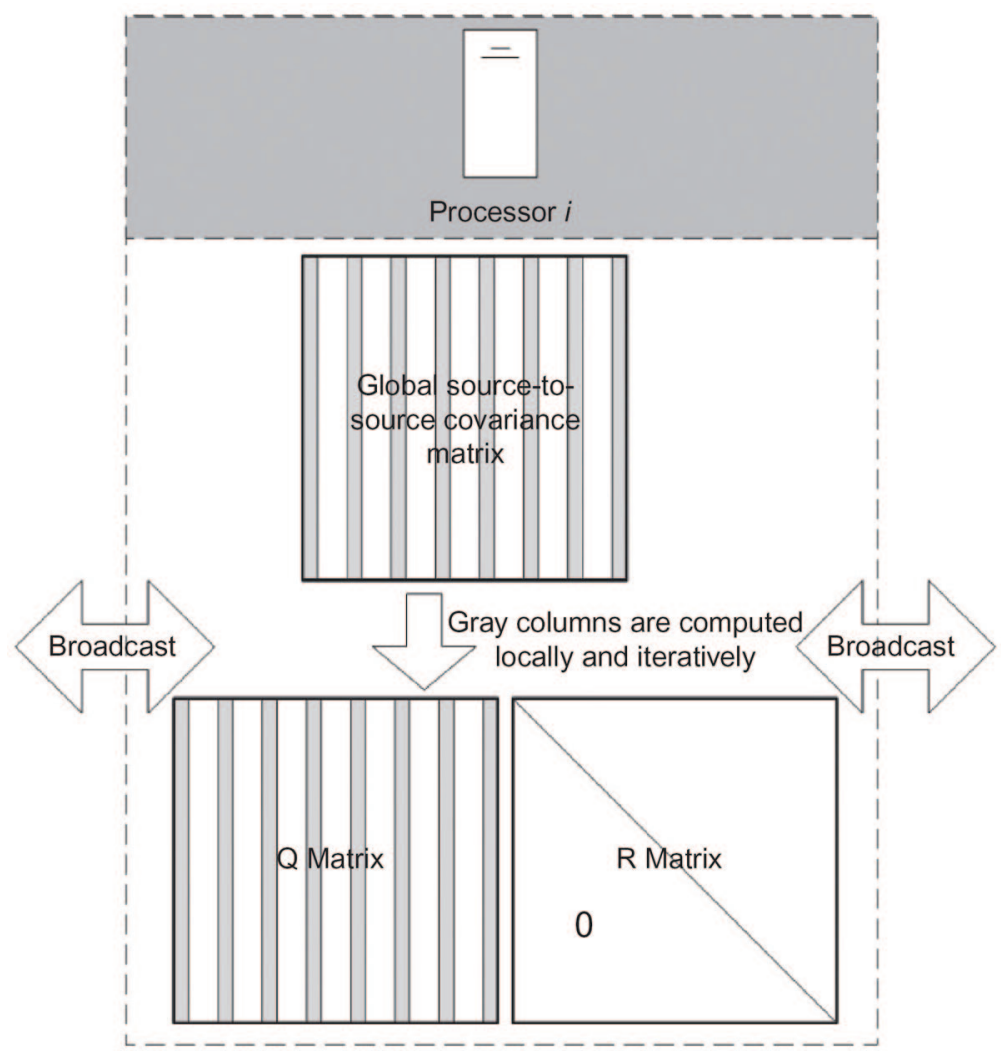

Figure 7. Detailed workflow of a processor for the second parallel process. 


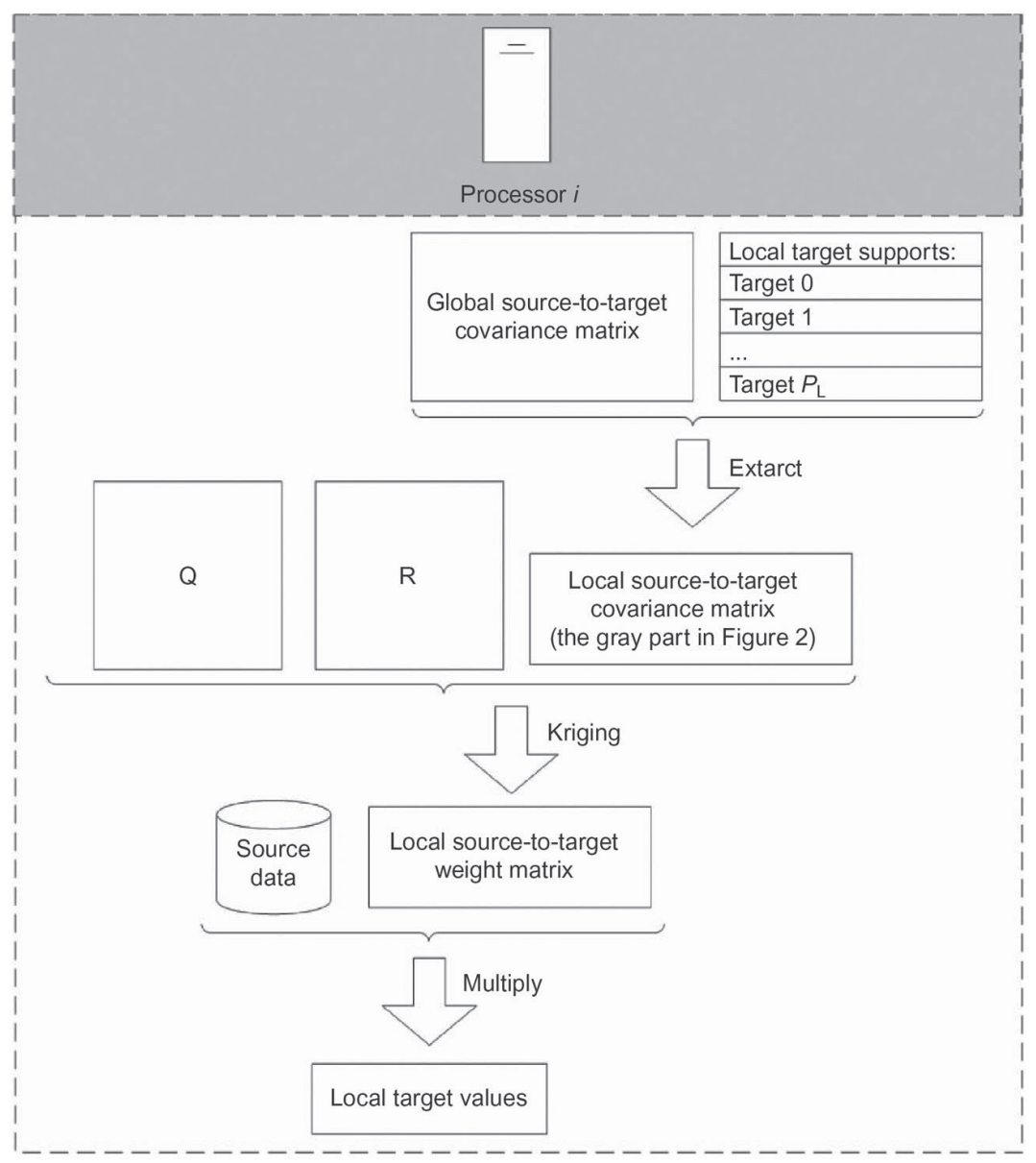

Figure 8. Detailed workflow of a processor for the third parallel process.

ization are quite mature and have been discussed extensively in the literature, this article will not elaborate on the details (for more information, see Cosnard and Robert 1986, Cosnard et al. 1986, Chu and George 1990). Note that unlike the other two parallel processes that do not require communication among processors during the computation, this parallel QR factorization process involves frequent data exchange along the iterations, which may become a major bottleneck of speed-up as the number of processors increases. To reduce the effect of communication overhead on the performance, the unblocking communication technique is used to overlap computation and data exchange (for technical details, see Gropp et al. 1998).

(8) Constructing the Kriging system and computing the local STT weight matrix and local target predictions (third parallel process, Figure 8)

According to its assignment in the target task map built in step 4, each processor extracts the covariance values from the global STT covariance matrix to form a local STT covariance matrix (the gray part in Figure 2). The local STT covariance matrix, together with the QR factorization of the STS covariance matrix, will be used to solve the Kriging system and obtain the local STT weights 
on each processor (see Section 1.2). The local target attribute predictions are then computed using the source attribute values and STT weights (Davis and Grivet 1984).

(9) Gathering target values and outputting

The master processor gathers the predicted target attribute values from all processors, re-orders them according to the target task map, and writes them back to the database.

\subsection{Implementation}

The parallel algorithm discussed above was implemented (see Appendix 1) using the C++ programming language and the Message Passing Interface (MPI), which is a standard parallel programming paradigm (Gropp et al. 1998) supported by almost all parallel computing systems. The FFT-based support-to-support covariance computation code was originally written by Liu et al. (2006) and was then modified to meet the requirements of this parallel algorithm. For the support-to-support covariance computation, two public-domain libraries were used: the FFTW ( http://www.fftw.org ) was used to perform the FFT processing and the GsTL ( http://pangea.stanford.edu/ nremy/GTL/ ) was used to compute the point covariance. For geospatial data input and output, the Shapefile C Library ( http://shapelib.maptools.org/) was used to read and write shape-files. For matrix manipulation and operation, the Template Numerical Toolkit (TNT, http://math.nist.gov/tnt/index.html ) was used to manage and process the matrices in memory, for example, the STS and STT covariance matrices, the STT weight matrix, and so on. A parallel QR factorization algorithm was implemented by modifying the source code of the TNT library.

Several $\mathrm{C}++$ classes were developed to manage the information used in the algorithm, for example, the covariogram model, the discretization scheme, and the task map. A suite of functions was also developed to transfer the information and data (e.g., shapefile objects and TNT matrices) among processors for parallel computing.

Besides the input shapefiles of source and target supports, the program also requires users to specify the discretization density (i.e., the cell size of the resultant raster) and to provide a file specifying the covariogram model of the underlying discretization points. Users are also allowed to choose the task-mapping scheme. Users can choose either simple Kriging or ordinary Kriging to compute the STT weights, but they have to provide the target mean if simple Kriging is chosen.

\section{Experiments and performance}

\subsection{Datasets and experiment design}

Two datasets were used in our experiments to test the computational performance and scalability (i.e., changes in performance when using different numbers of processors and dealing with different sizes of datasets) of the parallel areal interpolation program.

(1) Population densities in the Eastern Time Zone. The counties in the Eastern Time Zone (2248 polygons) served as the source supports, and their population densities (people/miles 2 ) in year 2000 as the known source data. The watersheds in the Eastern Time Zone (1633 polygons) served as the target supports and their population densities were to be predicted. 
(2) Population densities in the continental United States. The counties in the continental United States (4703 polygons) served as the source supports, and their population densities (people/miles ${ }^{2}$ ) in year 2000 as the known source data. The watersheds in the continental United States (3848 polygons) served as the target supports and their population densities were to be predicted.

A discretization scheme of 2000 meter cell size (i.e., node spacing) was used for both datasets, yielding a $1333 \times 917$ grid for the Eastern Time Zone dataset and a $1452 \times 2348$ grid for the continental US dataset. The size of the continental US dataset is significantly larger than that of the Eastern Time Zone dataset in all aspects, that is, the number of source supports (4703 vs. 2248), the number of target supports (3848 vs. 1633), and the number of discretization points (3.4 million vs. 1.2 million). The size of a dataset determines not only the amount of computing time but also the communication overhead, hence affects the performance, that is, speed-up and efficiency. Thus, the continental US dataset presents a much larger problem size to the areal interpolation algorithm than the Eastern Time Zone data does. Ideally, a well-designed and implemented parallel algorithm should scale well with the problem size, that is, the effect of problem size on the speed-up and efficiency is optimally minimized.

\subsection{Performance evaluation}

All experiments were conducted on a Linux cluster that consists of 1151 nodes, 280 of which have two AMD quad core processors (2.2 GHz, 8 GB RAM per node), the other 871 nodes have two dual core Opteron processors (2.8 GHz, 8 GB RAM per node). All nodes are connected with $800 \mathrm{MB} /$ second InfiniBand. The program was forced to run only on the Opteron nodes in order to minimize the effect of the variation of processors' computing speed. Note that the minimum computing unit in this cluster is the CPU core, and each core serves as an independent 'processor' with a maximum memory of $2 \mathrm{~GB}$. The following performance analysis will use the terms 'core' and 'processor' interchangeably.

The computing times were recorded for runs with different computing scenarios (e.g., input covariogram model, number of CPU cores, and task-mapping scheme). Since the variation of covariogram model does not cause significant change in the overall computational complexity, one can expect minimal effect on the performance to be introduced by such variation. The experiments also verified this hypothesis. Using different covariogram models, the average difference in computing time accounted for less than $2 \%$ of the overall time for the continental US dataset. Since the prediction accuracy and uncertainty are not the focus of this study, the performance analysis will be based on the experiments using a relatively simple spherical covariogram model, which was designed based on no empirical knowledge of the regions.

Also, because all task-mapping schemes used in this study distribute the same number of supports to the CPU cores, the workload distribution among cores is approximately the same for all mapping schemes. In fact, the experiments showed very little difference in computing time caused by task-mapping schemes. On average, only $3 \%$ of the total computing time for the continental US dataset was found different using different mapping schemes. Thus, the performance analysis will only focus on the results obtained using a particular task-mapping scheme, that is, the cyclic scheme. The prediction results using ordinary Kriging are shown in Figures 9 and 10.

Using only one CPU core, it took the program 3577.78 seconds (about 1 hour) to process the Eastern Time Zone dataset and 29341.48 seconds (more than 8 hours) to process 
(a)

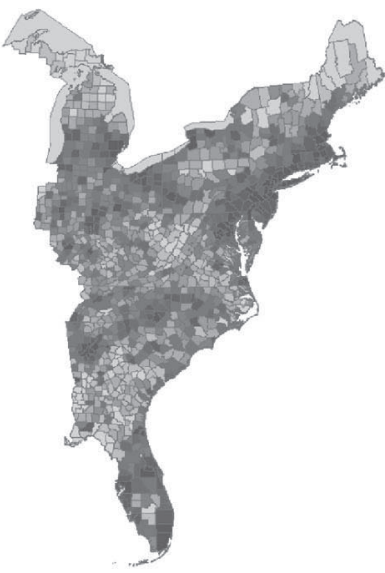

(b)

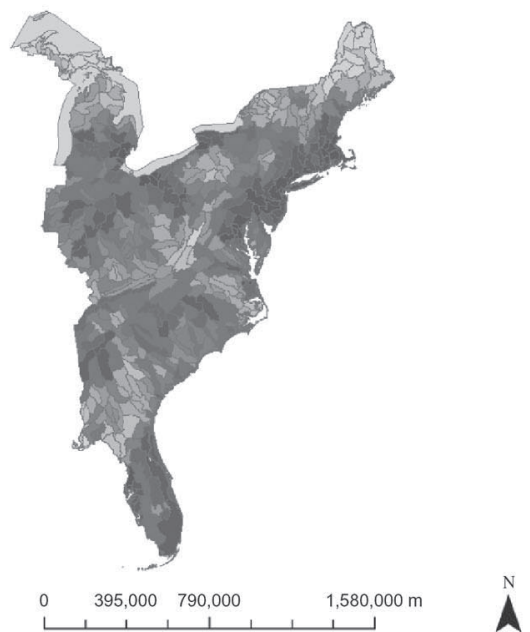

Figure 9. Predictions of population densities of watersheds in the Eastern Time Zone. (a) Population densities of counties and (b) population densities of watersheds.

the continental US dataset, which places this geostatistical areal interpolation at the very edge of feasibility in real-world applications. However, with more CPU cores utilized, the computing time was drastically reduced (Figure 11). When 512 cores were used, only 16.35 seconds were needed for the Eastern Time Zone dataset and 91.61 seconds for the continental US dataset, which can be considered reasonably practicable for actual geospatial analysis applications. Experiments also showed that the computing time for the sequential processes (e.g., support discretization and task mapping) remained almost static, and the communication and waiting time (besides the communication embedded in the second parallel process) slightly increased as the number of CPU cores increased. The sequential computation and communication/waiting only took a small fraction of the overall computing time when small numbers of CPU cores were used and occupied larger

(a)

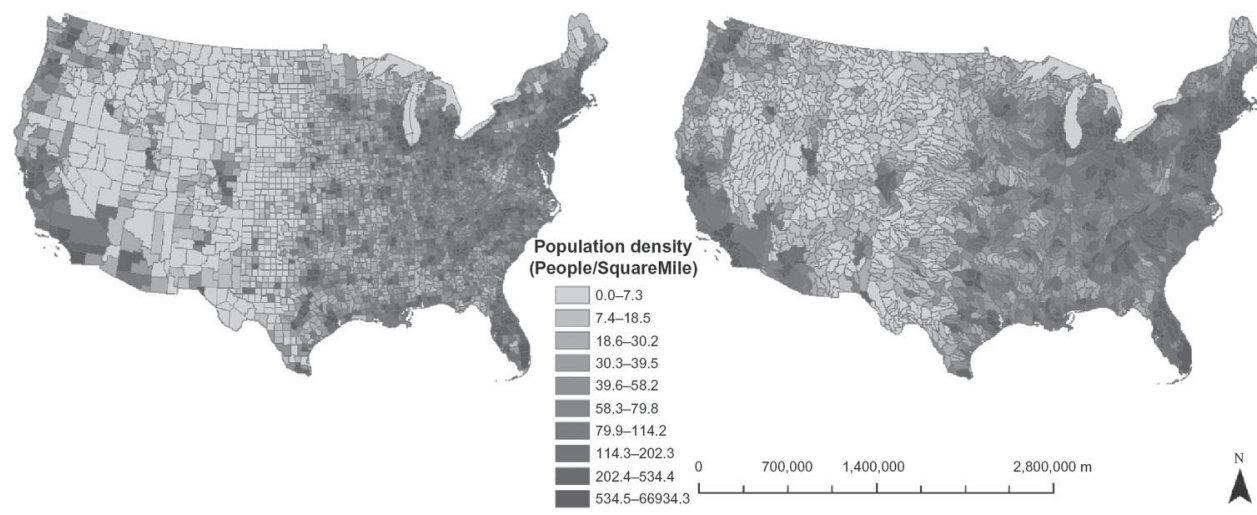

Figure 10. Predictions of population densities of watersheds in the continental United States. (a) Population densities of counties and (b) population densities of watersheds. 
(a)

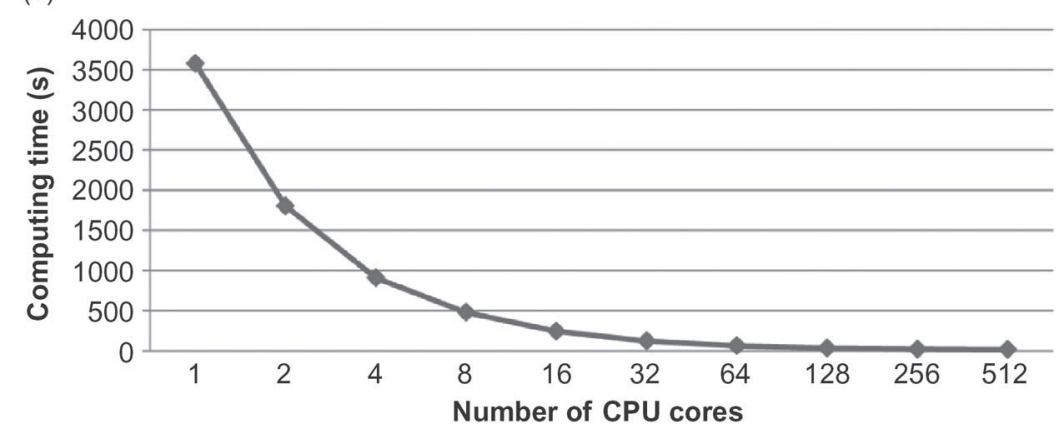

(b)

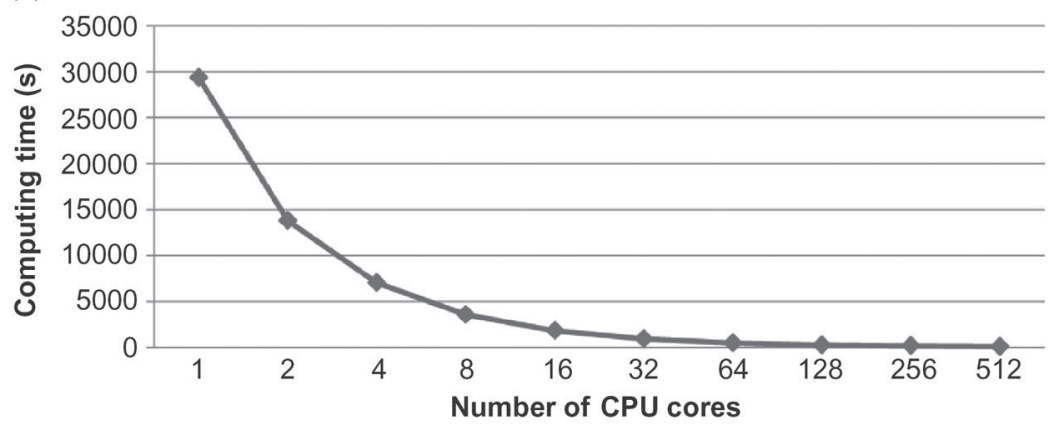

Figure 11. Computing times (cyclic task mapping). (a) Eastern Time Zone dataset and (b) continental US dataset.

proportion as the number of cores increased and the computing time for the parallel processes significantly decreased (Figure 12).

Figure 13 shows the speed-ups that the program and its three parallel processes achieved. Speed-up is one of the most frequently used criteria for assessing the performance of parallel programs. Considering an algorithm that executes on a parallel system with $p$ processors (i.e., CPU cores in this study) in time $t_{p^{\prime}}$ and the one that executes on a sequential system (with only one processor) in time $t_{1}$, the speed-up is defined as the following ratio:

$$
S_{p}=\frac{t_{1}}{t_{p}}
$$

In most cases, $1 \leq S_{p} \leq p$ (Cosnard and Trystram 1995). Note that $S_{p}=p$ is the ideal case (termed linear speed-up), which means the algorithm is perfectly parallelized and the workload is evenly distributed amount processors. Since most algorithms are not completely parallelizable, some of their parts have to be executed sequentially, and the communication between processors is usually inevitable, this ideal linear case is very hard to achieve. However, in some rare cases, super linear speed-up $\left(S_{p}>p\right)$ may occur. With the larger accumulated cache size when using multiple processors, more or even all dataset can fit into caches and the memory access time will be reduced dramatically, which yields extra speed-up in addition to that from the increased computing power. 

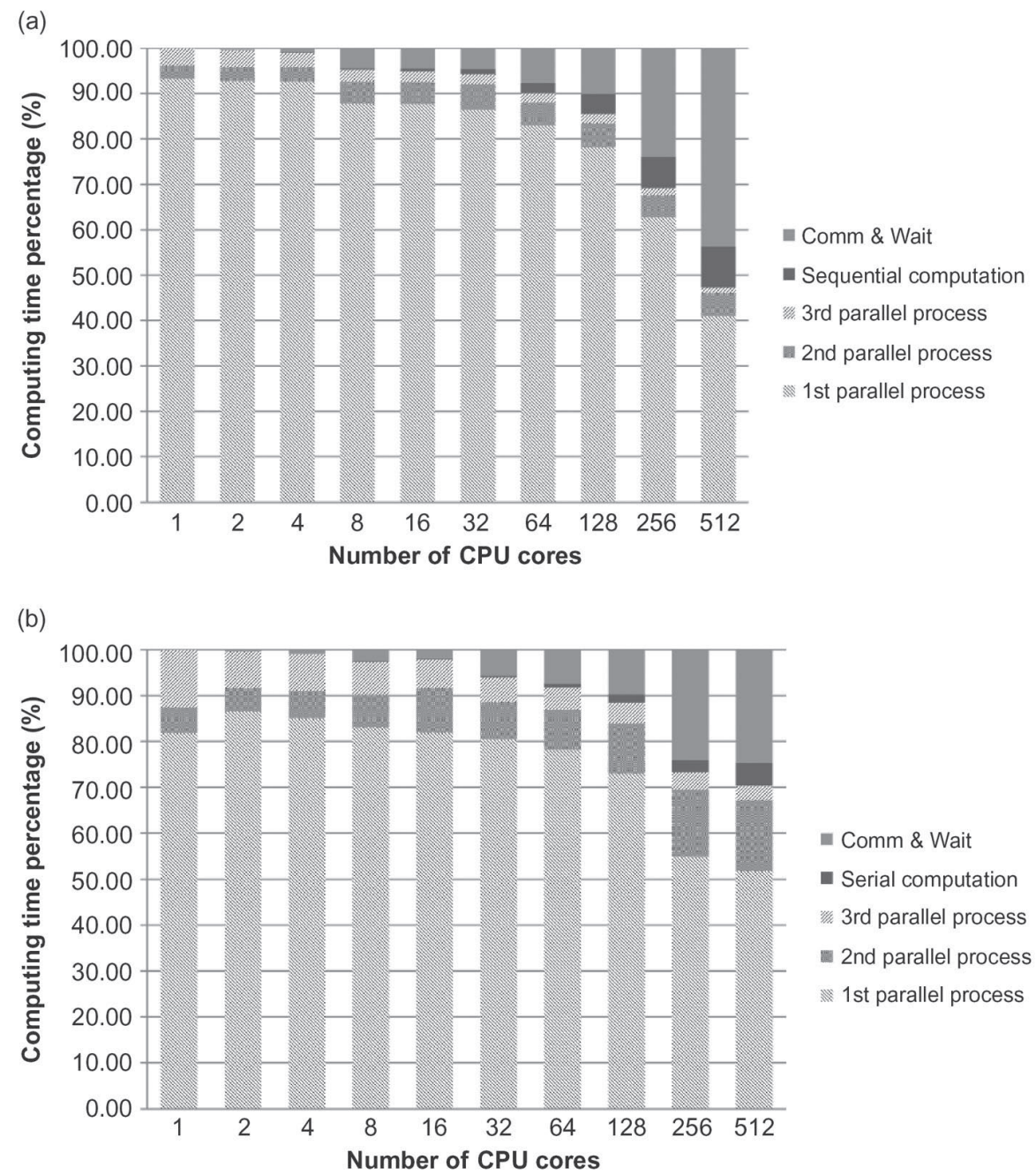

Figure 12. Computing time percentages (cyclic task mapping). (a) Eastern Time Zone dataset and (b) continental US dataset.

In this parallel areal interpolation program, the first parallel process (i.e., the FFTbased computation of support-to-support covariance matrices) constantly yielded near linear speed-ups for both datasets, due to its even distribution of workload among processors and minimal requirement for communication during the computation. In contrast, the second parallel process (i.e., the QR factorization) involves frequent data exchange during the computation (i.e., the broadcast of a completed column of matrix $Q$ at each iteration). In the experiments, the speed-ups of the second parallel process also demonstrated an increasing trend in general, but the pace of increase was significantly reduced by the increased cost of communication caused by larger number of processors (i.e., cores). The third parallel process (i.e., the computation of STT weights and target attribute values) yielded super linear speed-ups, most likely because of the reason mentioned above. The overall speed-ups, when relatively small numbers of CPU cores $(\leq 128)$ were used, were largely determined by the first parallel process for its dominating occu- 

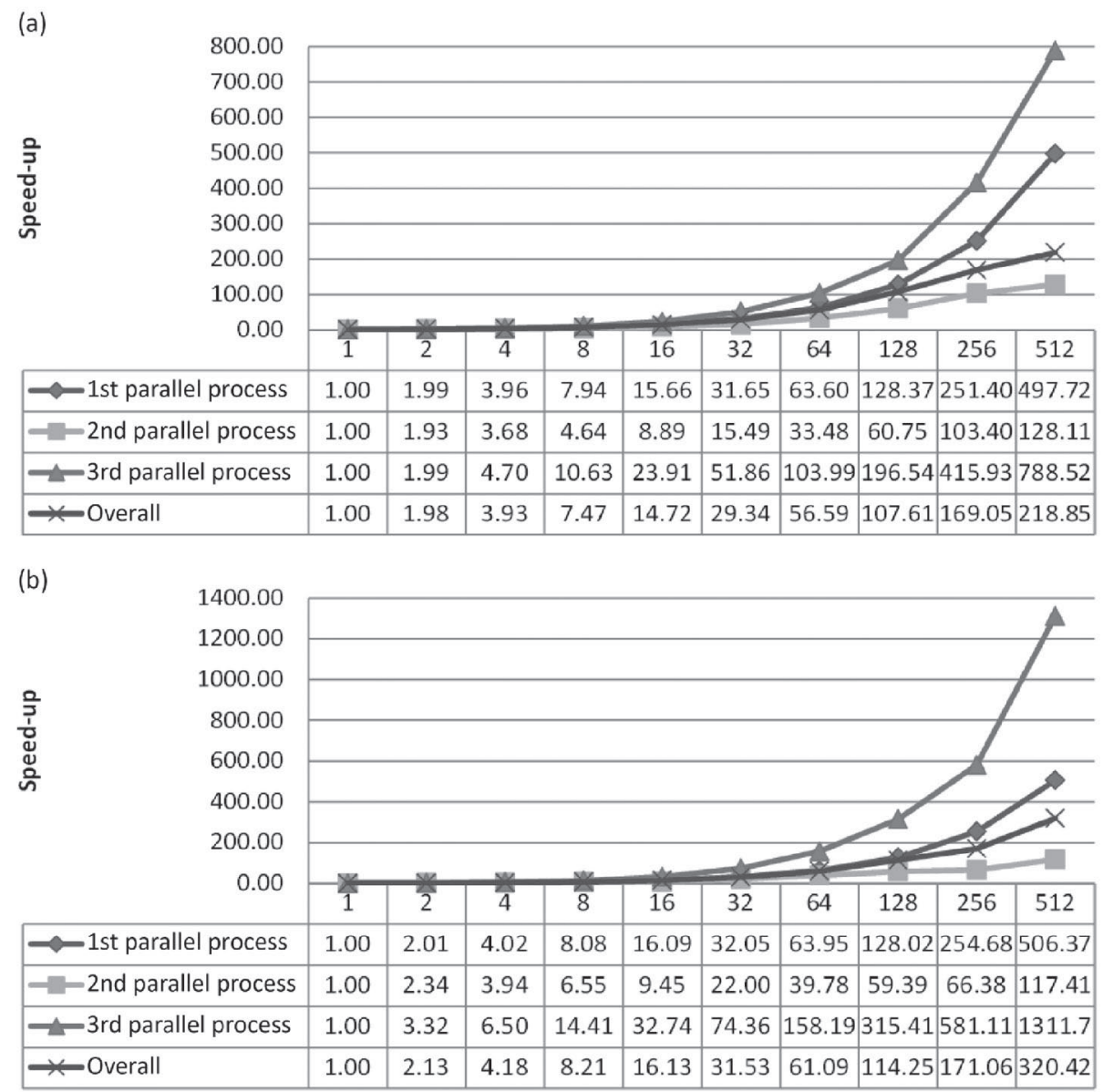

Figure 13. Speed-ups (cyclic task mapping). (a) Eastern Time Zone dataset and (b) continental US dataset.

pation of the overall computing time, and demonstrated a near linear increasing pattern. As larger numbers of cores were used $(\geq 256)$, the time proportion of the first parallel process (along with other two parallel processes) greatly decreased, and the sequential computation and communication increased their proportions in the overall computing time. Thus, the overall speed-ups slowed down and shifted away from the linear speed-up.

A comparison between the speed-ups for both datasets showed a similar increasing pattern in most cases, meaning this parallel program scales well for different problem sizes. The similarity remains until 512 CPU cores are used, when the computing time for sequential computation and communication outweighs the time for parallel processes for the Eastern Time Zone dataset, whereas the parallel processes still occupy the majority of the overall computing time for the continental US dataset (see Figure 12).

Figure 14 shows the efficiencies of the parallel program and its three parallel processes. Efficiency $e_{p}$ is calculated based on speed-up as follows:

$$
e_{p}=\frac{S_{p}}{p}
$$


(a) Eastern Time Zone Dataset

\begin{tabular}{|c|c|c|c|c|c|c|c|c|c|c|c|}
\hline \multirow{7}{*}{\multicolumn{2}{|c|}{ }} & \multicolumn{10}{|c|}{$\rightarrow$} \\
\hline & & \multirow{2}{*}{\multicolumn{10}{|c|}{$x+\infty$}} \\
\hline & & \multicolumn{9}{|c|}{$x \longrightarrow$} & \\
\hline & & \multicolumn{10}{|c|}{ 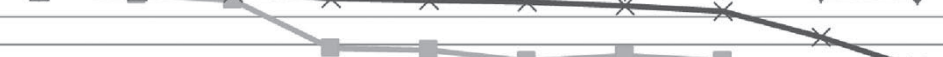 } \\
\hline & & \multirow{2}{*}{\multicolumn{10}{|c|}{ 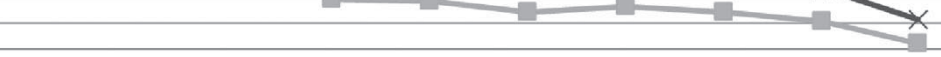 }} \\
\hline & & & & & & & & & & & \\
\hline & & 1 & 2 & 4 & 8 & 16 & 32 & 64 & 128 & 256 & 512 \\
\hline \multicolumn{2}{|c|}{$\multimap 1$ st Parallel Process } & 1.00 & 1.00 & 0.99 & 0.99 & 0.98 & 0.99 & 0.99 & 1.00 & 0.98 & 0.97 \\
\hline \multicolumn{2}{|c|}{-2 nd Parallel Process } & 1.00 & 0.97 & 0.92 & 0.58 & 0.56 & 0.48 & 0.52 & 0.47 & 0.40 & 0.25 \\
\hline \multicolumn{2}{|c|}{$\longrightarrow$ - 3rd Parallel Process } & 1.00 & 0.99 & 1.17 & 1.33 & 1.49 & 1.62 & 1.62 & 1.54 & 1.62 & 1.54 \\
\hline \multicolumn{2}{|c|}{ —Overall } & 1.00 & 0.99 & 0.98 & 0.93 & 0.92 & 0.92 & 0.88 & 0.84 & 0.66 & 0.43 \\
\hline
\end{tabular}

(b) Continental U.S. Dataset

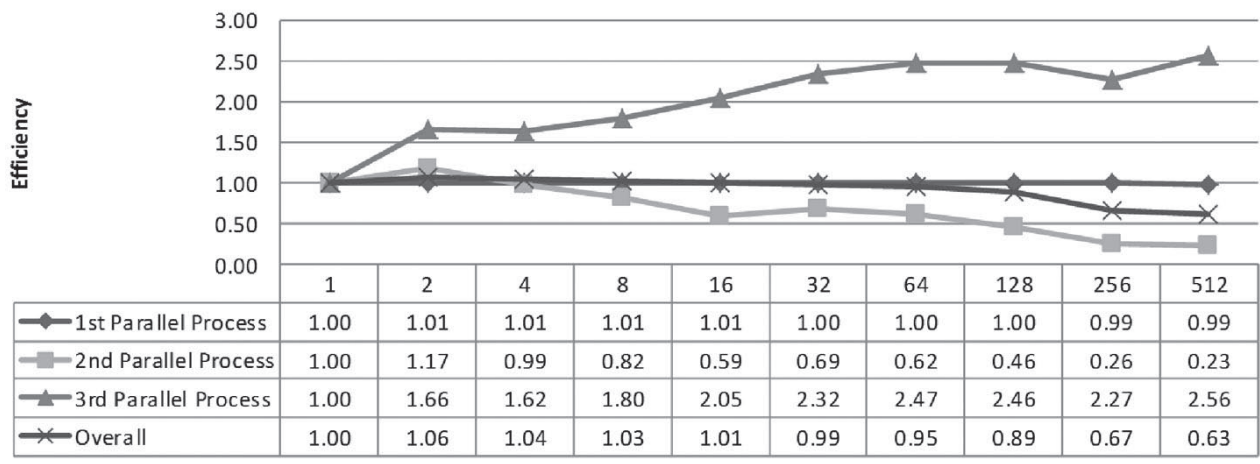

Figure 14. Efficiencies (cyclic task mapping). (a) Eastern Time Zone dataset and (b) continental US dataset.

Efficiency is used to measure the average utilization of processors. In most cases, $e_{p} \leq 1$. The better the parallelism of the algorithm, the nearer $e_{p}$ is to 1 . When linear speedup is achieved, $e_{p}$ equals to 1 . Generally, efficiency declines as the number of processors increases, because the communication overhead increases.

For both datasets, the efficiency of the first parallel process remained nearly static around 1; the efficiency of the second parallel process dropped quickly as the number of cores increased because of the embedded communication overhead; and the efficiency of the third parallel process stayed above 1 for its super linear speed-up. The overall efficiency for both datasets gradually dropped down as the number of CPU cores increased, but stayed above 0.8 in most cases until the number of cores reached 128.

\section{Conclusion and discussion}

Geostatistical areal interpolation based on variants of Kriging remains extremely computationally intensive, requiring extensive amount of memory space and massive computing power. Little work has been done to employ parallel computing technology to tackle such computational obstacle. This study parallelizes the FFT/Kriging-based areal interpolation algorithms and attempts to promote its usage in real-world spatial analysis applications. 
The parallel areal interpolation algorithm developed in this study includes three parallel processes: (1) the FFT processing for the source supports and the computation of STS and STT covariance matrices; (2) the QR factorization of the STS covariance matrix; (3) the computation of STT weights via Kriging and the computation of attribute values for the target supports. The implementation of this parallel algorithm utilizes the general-purpose MPI library and other public-domain programming libraries, thus ensuring the portability of the algorithm across a large variety of parallel computing systems.

Experiments using real-world datasets showed that this parallel algorithm drastically reduced the computing time, achieved fairly high speed-ups and efficiencies, and scaled reasonably well as the number of processors increased and as the problem size increased. With the increasingly wide adoption of high-performance computing technologies, including massive supercomputers, computer clusters, multi-core computers, distributed computing, and the recently emerging Cyberinfrastructure (i.e., Grid computing and Cloud computing), users are able to perform such geostatistical areal interpolation with vast volume of geospatial datasets, which used to be infeasible for real-world geospatial applications in terms of memory space and computing time, within a practically short period of time. Also, the parallel program developed in this study can be easily added to an existing ArcGIS toolbox developed by Schneider and Kyriakidis (2006), which provides a graphical user interface for geostatistical areal interpolation. To do so, a link to a MPI-based parallel computing environment (e.g., a local multi-core computer or a remote high-performance computing system) needs to be established, which can be implemented using a Python wrapper.

The parallel algorithm developed in this study is based on global Kriging, which uses attribute values at all available source supports to predict the attribute value of a particular target support. To this respect, (global) area-to-point Kriging or downscaling (Kyriakidis 2004, Yoo et al. 2010) would benefit from the parallel computing speed-ups achieved in this work. Nevertheless, global Kriging is more computationally expensive and requires more memory space and computing time compared to local Kriging (Goovaerts 1997, Chilès and Delfiner 1999). Even though this parallelization approach can be adapted to local Kriging, an efficient neighbor-searching process will be needed.

Also, this study uses regular discretization grids for support-to-support covariance computation, because FFT-based technology can only be used when covariance integrals are discretized with such regular schemes (Chilès and Delfiner 1999). Evidently, irregular discretization grids may be used in some cases by specifying scattered locations at which the point covariance model will be evaluated. For irregularly shaped areal units, this would result in fewer discretization points and lower computational intensity (Goovaerts 2008), as compared with a regular grid attempting to adequately discretize complex geometrical shapes. Nevertheless, the parallelization approach used in this study will still stand for geostatistical areal interpolation with irregular discretization grids. Since supports may be discretized using different schemes, the dimensions of the sampling function vector (hence the number of discretization points $N$ ) will no longer be uniform as in regular grid cases, thus the computational complexity for the support-to-support covariance may vary across supports. Therefore, a discretizationbased task-mapping scheme is needed to optimally distribute the workload to processors. Static task-mapping heuristics (Braun et al. 2001) can be used in this case because the computational intensity can be estimated based on the number of discretization points. 


\section{Notes}

1. A global matrix refers to a matrix that includes all support-to-support pairs. For example, a global STT covariance matrix includes the covariance values between all source and all target supports.

2. Local supports refer to the supports assigned to a particular processor.

\section{References}

Armstrong, M. P. and Marciano, R., 1993. Parallel spatial interpolation. In: Proceedings of the eleventh international symposium on computer-assisted cartography (Auto-Carto 11). Bethesda, MD: American Congress on Surveying and Mapping, 414-423.

Armstrong, M. P. and Marciano, R., 1994. Inverse distance weighted spatial interpolation using a parallel supercomputer. Photogrammetric Engineering and Remote Sensing, 60 (9), 1098-1104.

Armstrong, M. P. and Marciano, R., 1995. Massively parallel processing of spatial statistics. International Journal of Geographical Information Science, 9 (2), 169-189.

Armstrong, M. P. and Marciano, R. J., 1996. Local interpolation using a distributed parallel supercomputer. International Journal of Geographical Information Systems, 10 (6), 713-729.

Armstrong, M. P. and Marciano, R. J., 1997. Massively parallel strategies for local spatial interpolation. Computers \& Geosciences, 23 (8), 859-867.

Braun, T., et al., 2001. A comparison of eleven static heuristics for mapping a class of independent tasks onto heterogeneous distributed computing systems. Journal of Parallel and Distributed Computing, 61 (6), 810-837.

Chilès, J. and Delfiner, P., 1999. Geostatistics: modeling spatial uncertainty. New York: Wiley.

Chu, E. and George, A., 1990. QR factorization of a dense matrix on a hypercube multiprocessor. SIAM Journal on Scientific and Statistical Computing, 11 (5), 990-1028.

Cosnard, M., Muller, J.-M., and Robert, Y., 1986. Parallel QR decomposition of a rectangular matrix. Numerische Mathematik, 48 (2), 239-249.

Cosnard, M. and Robert, Y., 1986. Complexity of parallel QR factorization. Journal of ACM, 33 (4), 712-723.

Cosnard, M. and Trystram, D., 1995. Parallel algorithms and architecture. Boston, MA: International Thomson Computer Press.

Davis, M. W. and Grivet, C., 1984. Kriging in a global neighborhood. Journal of the International Association for Mathematical Geology, 16 (3), 249-265.

Frigo, M., 1999. A fast Fourier transform compiler. AGM SIGPLAN Notices, 34 (5), 169-180.

Gajraj, A., Joubert, W., and Jones, J., 1997. A parallel implementation of kriging with a trend. Los Alamos, NM: Los Alamos National Lab.

Goodchild, M. F., Anselin, L., and Deichmann, U., 1993. A framework for the areal interpolation of socioeconomic data. Environment and Planning A, 25 (3), 383-397.

Goodchild, M. F. and Lam, N. S. N., 1980. Areal interpolation: a variant of the traditional spatial problem. Geo-Processing, 1 (3), 297-312.

Goovaerts, P., 1997. Geostatistics for natural resources evaluation. New York: Oxford University Press.

Goovaerts, P., 2008. Kriging and semivariogram deconvolution in the presence of irregular geographical units. Mathematical Geosciences, 40 (1), 101-128.

Grama, A., et al., 2003. Introduction to parallel computing. New York: Addison-Wesley.

Gropp, W., et al., 1998. MPI: the complete reference. Vol. 2. Cambridge, MA: The MIT Press.

Haining, R., 2003. Spatial data analysis: theory and practice. Cambridge: Cambridge University Press.

Hawick, K. A., Coddington, P., and James, H., 2003. Distributed frameworks and parallel algorithms for processing large-scale geographic data. Parallel Computing, 29 (10), 1297-1333.

Karniadakis, G. and Kirby, R., II., 2003. Parallel scientific computing in C and MPI: a seamless approach to parallel algorithms and their implementation. New York: Cambridge University Press.

Kerry, K. E. and Hawick, K. A., 1998. Kriging interpolation on high-performance computers. In Proceedings of the international conference and exhibition on high-performance computing and networking. Berlin: Springer-Verlag, 429-438. 
Kyriakidis, P. C., 2004. A geostatistical framework for area-to-point spatial interpolation. Geographical Analysis, 36 (3), 259-289.

Kyriakidis, P. C. and Goodchild, M. F., under review. A geostatistical perspective on cartographic methods of areal interpolation. International Journal of Geographical Information Science.

Kyriakidis, P. C., Schneider, P., and Goodchild, M. F., 2005. Improving spatial data interoperability using geostatistical support-to-support interpolation. In: Proceedings of geoComputation. Ann Arbor, MI: University of Michigan.

Liu, Y., Jiang, Y., and Kyriakidis, P., 2006. Calculation of average covariance using fast Fourier transform $(F F T)$. Menlo Park, CA: Stanford Center for Reservoir Forecasting, Petroleum Engineering Department, Stanford University.

Morrison, J., 2000. Kriging in a parallel environment. In: Proceedings of geomatics for informed decisions (GEOIDE). Calgary, Alberta.

Pedelty, J., et al., 2003. High performance geostatistical modeling of biospheric resources in the Cerro Grande Wildfire Site, Los Alamos, New Mexico and Rocky Mountain National Park, Colorado. In: Proceedings of earth science technology conference (ESTC). College Park, MD: University of Maryland

Rossini, A. J., Tierney, L., and Li, N., 2007. Simple parallel statistical computing in R. Journal of Computational \& Graphical Statistics, 16 (2), 399-420.

Schneider, P. and Kyriakidis, P. C., 2006. Improving spatial support interoperability in GIS using geostatistics: An areal interpolation toolbox. In: Proceedings of the 4th international conference on geographic information science. Münster, Germany: GIScience.

Wang, S. and Armstrong, M. P., 2003. A quadtree approach to domain decomposition for spatial interpolation in grid computing environments. Parallel Computing, 29 (10), 1481-1504.

Yoo, E. H. and Kyriakidis, P. C., 2008. Area-to-point prediction under boundary conditions. Geographical Analysis, 40 (4), 355-379.

Yoo, E., Kyriakidis, P. C., and Tobler, W., 2010. Reconstructing population density surfaces from areal data: a comparison of Tobler's pycnophylactic interpolation method and area-to-point kriging. Geographical Analysis, 42 (1), 78-98.

Appendix 1. Introduction to the Supplementary Materials Parallel Geostatistical Areal Interpolation Source Code and Test Data

\section{Code Structure}

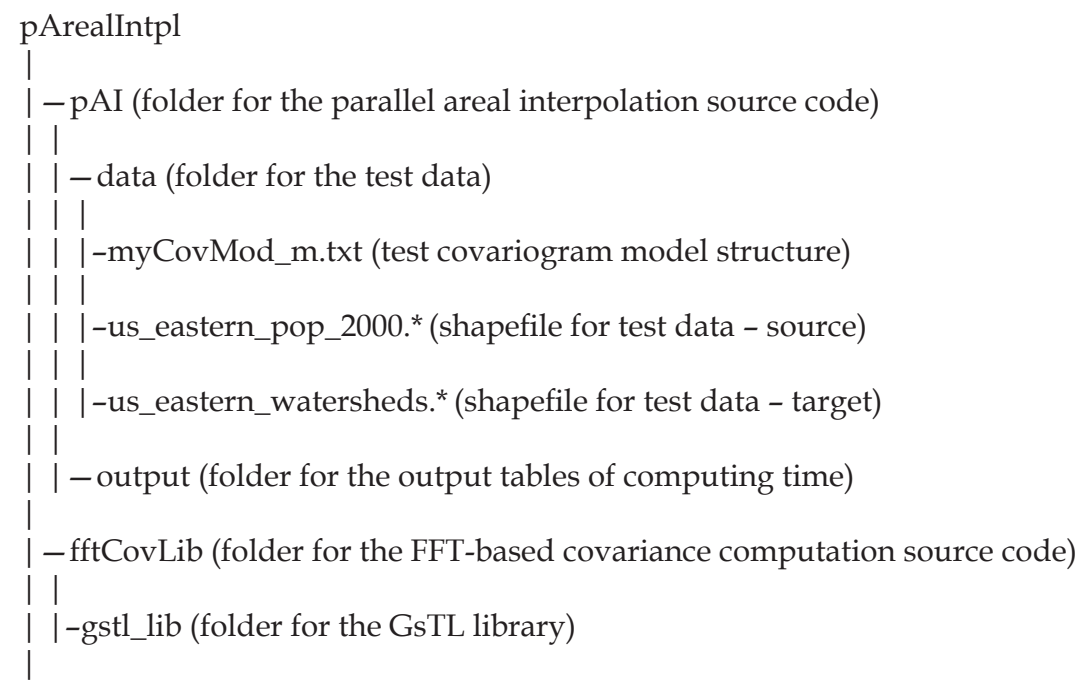


I - tnt (folder for the TNT library)

- shpLib (folder for the Shapefile C library)

| -ibcast (folder for the unblocking broadcast source code)

\section{Compile}

Note: This program has been tested on a CentOS 5.5 Linux system using OpenMPI 1.3.3, FFTW 3.2.2, and GCC 4.1.2

(1) Make sure MPI and the FFTW library is installed properly

(2) Change directory to $\mathbf{p A I}$ by typing ' $c d p A I^{\prime}$

(3) Open Makefile and modify the lines that specify the locations of MPI and FFTW

(4) Type 'make depend'

(5) Type 'make' to compile.

After successful compiling, an executable file named 'pAI' will be generated.

\section{Covariogram model file}

A covariogram model can include a nugget and multiple structures, and it must be organized as follows:

nugget num_structures

structure_type1 sill1 [ range11 range12 range13] [ angle11 angle12 angle13]

structure_type1 sill2 [ range21 range22 range23] [ angle21 angle22 angle23]

$\cdots$

where structure_type can be 'Spherical', 'Gaussian', or 'Exponential'.

For areal interpolation (i.e., 2D), only rang1, rang2, and angle1 need to be specified. Other ranges and angles can be set to 0 .

IMPORTANT: Angles must be in Radian.

Example:

01

Spherical 1 [ 50003000 0] [ 000 ] 


\section{Usage}

To run the program in a parallel computing environment:

mpirun -np $<$ num_proc $>p A I-i d<$ timefile_id $>-c f<$ covariance_filename $>-s f$
$<$ source_shpfilename $>-s a<$ source_attribute $>[-s i / s v]-t f<$ target_shpfilename $>-t a$
$<$ target_attribute $>[-t i / t v]-c s<c e l l \_s i z e>[-o k /-s k<$ target_mean $>][-b d /-c d /-r d m /-$
$b c d<$ number_of_blocks $>/-c b d<$ number_of_blocks $>][-q r /-l u]$

num_proc: number of processors/CPU cores timefile_id: a unique ID for the time log file covariance_filename: covariogram model file, in ASCII format source_shpfilename: shapefile name for the source supports source_attribute: source attribute field name -si: use this if the source attribute is extensive, for example, population -sv: use this if the source attribute is intensive, for example, population density target_shpfilename: shapefile name for the target supports target_attribute: target attribute field name $-t i$ : use this if the target attribute is extensive $-t v$ : use this if the target attribute is intensive cell_size: cell size for discretization, that is, grid spacing $-o k$ : use this if ordinary Kriging is to be used (default) -sk: use this if simple Kriging is to be used Note: The target mean MUST be specified. target_mean: mean value of target attributes $-b d$ : block mapping scheme (default) $-c d$ : cyclic mapping scheme $-r d m$ : random mapping scheme $-b c d$ : block-cyclic mapping scheme. The number of blocks MUST be specified. -cbd: cyclic-block mapping scheme. The number of blocks MUST be specified. number_of_blocks: must be specified when -bcd or -cbd is used. -qr: QR factorization. Parallelized (default) -lu: LU factorization. NOT parallelized

Example:

mpirun -np 32 pAI -id US_PopDensity -cf./data/myCovMod_m.txt-sf./data/ us_eastern_pop_2000.shp -sa POP00SQMIL -sv -tf./data/us_eastern_watersheds.shp -ta POPSQMIL -tv -cs 2000 -ok-cd -qr 